\title{
Editorial
}

Monatsschr Kinderheilkd 2021 · 169:1-2 https://doi.org/10.1007/s00112-020-01089-2

(c) Springer Medizin Verlag GmbH, ein Teil von Springer Nature 2020

Liebe Kolleginnen und Kollegen,

es ist mir eine Freude, in dieser Ausgabe der Monatsschrift Kinderheilkunde erstmals als Präsident der Deutschen Gesellschaft für Kinder- und Jugendmedizin (DGKJ) das Wort an Sie zu richten. Und es ist eine Ehre, der ersten Präsidentin der DGKJ im Amt folgen zu dürfen.

Das wichtigste Anliegen ist es, kontinuierlich den Prozess weiterzuentwickeln, der unter der enorm erfolgreichen Präsidentschaft von Frau Prof. KrägelohMann in den letzten 3 Jahren verantwortet wurde. Es ist daher nicht nur beruhigend, sondern auch zielführend, dass diejenigen, die über die letzten 2 Jahre zusammengearbeitet haben, für ein weiteres Jahr als Geschäftsführender Vorstand der DGKJ aktiv sind, allen voran Frau Prof. Krägeloh-Mann, flankiert vom Schatzmeister, Herrn Prof. Christian von Schnakenburg, dem Sprecher der Konventgesellschaften, Herrn Prof. Dominik Schneider, und dem Generalsekretär der DGKJ, Herrn PD Dr. Burkhard Rodeck.

Dieses Team wird unterstützt von einer professionellen, konstruktiven und fleißigen Geschäftsstelle unter Leitung von Frau Dr. Noleppa und Frau Dr. Olbrisch. Tragende Aufgaben im Team der Geschäftsstelle haben Frau Kohlos, Frau Kühne, Frau Dr. Lunau, Frau Marter und Frau Schmidt. Insofern ist gewährleistet, dass nicht alle 3 Jahre in der DGKJ eine neue Aufstellung erfolgen muss, sondern, im Gegenteil, wir unsere Gesellschaft mit einem erfahrenen und bekannten Team weiter nach vorne bringen können. Auch die ehrenamtliche Arbeit unserer Kom-

Jörg Dötsch

Klinik und Poliklinik für Kinder- und Jugendmedizin, Uniklinik Köln, Köln, Deutschland

\section{Konstruktive Zusammenarbeit und nachwuchsorientierte Zukunftsgestaltung in der Kinder- und Jugendmedizin}

missionen soll an dieser Stelle gewürdigt werden.

Ein besonderes Merkmal der $\mathrm{Ge}$ schäftsstelle ist, dass sie sich gemeinsam mit wichtigen pädiatrischen Verbänden in Berlin auf dem gleichen Flur befindet. Dort findet sich eine Vertretung des Berufsverbandes der Kinder- und Jugendärzte (BVKJ), die Deutsche Akademie für Kinder- und Jugendmedizin (DAKJ), die Gesellschaft der Kinderkrankenhäuser und Kinderabteilungen in Deutschland (GKinD), die Deutsche Gesellschaft für Sozialpädiatrie (DGSPJ) und weitere Konventgesellschaften.

\section{\) Die Vielfalt in der Pädiatrie müssen wir als unsere Stärke verstehen}

Dies bringt mich zu dem ersten wichtigen inhaltlichen Thema, der verbändeübergreifenden Zusammenarbeit innerhalb der Pädiatrie. Dieses Thema ist essenziell, da ein nicht oder wenig abgestimmtes Vorgehen der pädiatrischen Verbände insbesondere in Politik oder Öffentlichkeit Verwirrung über die Position der Kinder- und Jugendmedizin auslösen kann. Nichts wäre verheerender als der Eindruck, die wesentlichen kinder- und jugendmedizinischen Ziele unterschieden sich in Abhängigkeit von der Zugehörigkeit zu einem Verband. Daher liegt ein ganz großes Interesse für uns im Vorstand der DGKJ darin, die verbändeübergreifende Zusammenarbeit zu modernisieren und neu zu gestalten. Wir haben das Ziel, neue innovative Formate einzuführen, die es uns erlauben, mit den Mitteln der modernen Informationstechnologie Kommunikation schneller und transparenter zu gestalten. Ganz wichtig ist es uns, alle wesentlichen Verbände in der Kinder- und Jugendmedizin zusammenzubringen und den Abstimmungsprozess untereinander so gründlich wie möglich zu gestalten.

Natürlich heißt dies nicht, dass es nicht mitunter zu Meinungsverschiedenheiten kommt. Völlig natürlich ist das Interesse einer/eines unternehmerisch verantwortlichen ambulant tätigen Kollegin/Kollegen zumindest in Teilen anders als das der/des fest angestellten Klinikärztin/-arztes. Vorschlag zur Ergänzung: Auch sind die Schwerpunktsetzungen der in der Pädiatrie vertretenen Spezialdisziplinen sehr unterschiedlich. Insofern sind Meinungsaustausch sowie die Akzeptanz unterschiedlicher Interessen und diese, wo immer möglich, auszugleichen, ein wesentliches Moment unserer Arbeit. Die Vielfalt in der Pädiatrie müssen wir als unsere Stärke verstehen. Es ist daher notwendig, entstehende Konflikte auszutragen und auch auszuhalten, immer mit dem Blick auf eine gemeinsame konstruktive Lösung.

Ein weiteres wichtiges Augenmerk wird die weitere Verbesserung der Stellung der Kinder- und Jugendmedizin in der Gesellschaft im Allgemeinen, v.a. aber im Hinblick auf ihre Finanzierung im Konzert der anderen Interessenverbände des Gesundheitssystems sein. Die laufenden Gespräche mit Ministerien, den Beauftragten der Bundesregierung, den Ausschüssen und Kommissionen 
des Bundestages und den Abgeordneten sowie den Spitzenverbänden der Krankenkassen und dem Gemeinsamen Bundesausschuss werden fortgeführt. Eine besondere Rolle spielen dabei die Arbeit gegen die Fehlentwicklungen im Hinblick auf die Kinder- und Jugendmedizin, wie die fehlende Bereitstellung von Vorhaltekosten und die inadäquate Finanzierung von pädiatrischen Ambulanzen/Tageskliniken, sowie auch der konstruktive Umgang mit besonders hohen kostenintensiven Patienten. Wichtig ist auch hier ein guter und partnerschaftlicher Abgleich der Interessen mit allen Sektoren der pädiatrischen Versorgung.

Eine besondere Bedrohung der Versorgung entsteht durch den Fachkräftemangel im pflegerischen und auch, in bestimmten Teilen der Pädiatrie, im ärztlichen Bereich. Auch hier wird es in den nächsten Jahren von entscheidender Bedeutung sein, auf der einen Seite die Politik und die Öffentlichkeit mit ins Boot zu nehmen, wenn es darum geht, Lösungen zu finden. Auf der anderen Seite müssen die Attraktivität unseres Faches und die Freude, die mit der Arbeit mit Kindern und Jugendlichen verbunden ist, an die jüngere Generation weitergegeben werden, um engagierten und optimistischen Nachwuchs in die pflegerische und ärztliche Kinder- und Jugendmedizin zu bringen.

Eine große Errungenschaft der Amtszeit von Frau Prof. Krägeloh-Mann, vorbereitet durch ihre Vorgänger, war die Einrichtung des Deutschen Zentrums für Kinder- und Jugendgesundheit, einer institutionalisierten pädiatrischen Einrichtung für die wissenschaftliche Weiterentwicklung unseres Faches. Von erheblicher Bedeutung ist nun die Ausstattung dieses Zentrums in einer ähnlichen Dimension wie bei den bestehenden deutschen Gesundheitszentren. Entscheidend ist, dass wir das Interesse des Nachwuchses für die wissenschaftliche Arbeit wecken. Von besonderer Relevanz wird es sein, alle Sektoren mit in die Forschungsaktivitäten der Kinderund Jugendmedizin $\mathrm{zu}$ integrieren und sie so weit $\mathrm{zu}$ führen, dass hieraus im Idealfall allgemeingültige pädiatrische Präventionsstrategien entstehen.
Die herausragende Aufgabe der Förderung unseres Nachwuchses ist mehrfach zum Ausdruck gekommen. Hierfür ist die Weiterentwicklung unserer Fortund Weiterbildung von entscheidender Bedeutung. Dies gelingt immer da leichter, wo gemeinsame Aktivitäten entfaltet werden. So planen wir, Fortbildungsveranstaltungen in stärkerer Kooperation mit den anderen pädiatrischen Verbänden abzubilden und auch in diesem Bereich vermehrt auf die Beteiligung jüngerer Kinder- und Jugendärztinnen und Kinder- und Jugendärzte zu setzen sowie moderne Informationstechnologien gerade in Pandemiezeiten zur Anwendung zu bringen. Diese Aktivitäten bieten sich darüber hinaus an, wann immer es um internationale Vernetzung mit europäischen oder außereuropäischen Pädiatrien geht.

Als weitere wichtige Themen möchte ich beispielhaft die Prävention und die Arzneimittelversorgung in der Kinderund Jugendmedizin nennen.

$\mathrm{Zu}$ guter Letzt soll auch das wichtige Thema der pädiatrischen Zeitschriften erwähnt sein. Die vor Ihnen liegende $M o$ natsschrift Kinderheilkunde ist eines der wichtigsten und hochgeschätzten Medien, über die die Kinder- und Jugendmedizin in den deutschsprachigen Ländern miteinander kommuniziert. Dies zu flankieren von einer stärker wissenschaftlich orientierten Online-Zeitschrift, der Molecular Cellular Pediatrics, ist seit einigen Jahren unser großes Ziel, und wir hoffen, in den kommenden Jahren den Durchbruch bei der Etablierung zu erreichen.

Ich möchte Sie, liebe Kolleginnen und Kollegen, sehr herzlich einladen, mit uns in der Geschäftsstelle oder im Vorstand direkt in Kontakt zu treten, Ihre Ideen, Anregungen, aber auch Kritikpunkte uns gegenüber zum Ausdruck zu bringen, damit es auch weiterhin gelingt, dass wir gemeinsam die Pädiatrie in Deutschland weiterentwickeln und ihr das Erscheinungsbild geben, das ihr gebührt: das des schönsten Faches in der Medizin. Oder wie die junge DGKJ sagen würde: ... mit der besten „work-smile balance“.

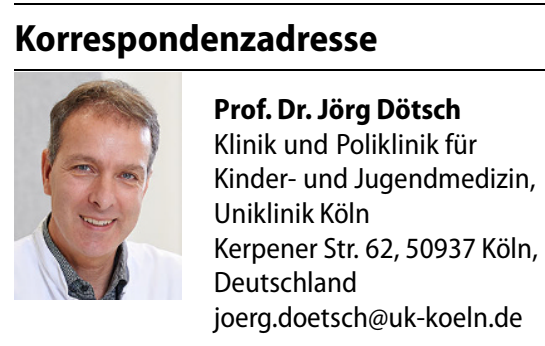

Interessenkonflikt. J. Dötsch gibt an, dass kein Interessenkonflikt besteht. 